Quantitative Political Economy Research Group

Department of Political Economy

King's College London

\title{
QPE
}

\section{Spreading the disease: The role of culture}

QPE Working Paper 2020-8

Ioannis Laliotis

Dimitrios Minos

June 15,2020 


\title{
Spreading the disease: The role of culture
}

\author{
Ioannis Laliotis* Dimitrios Minos ${ }^{\dagger}$
}

June 14, 2020

\begin{abstract}
This paper investigates the "cultural" transmission of the SARS-CoV-2 outbreak. Using West Germany data we observe that in predominantly Catholic regions with stronger social and family ties, the spread and the resulting deaths per capita were much higher compared to non-Catholic ones at the NUTS-3 level. This finding could help explain the rapid spread and high death toll of the virus in some European countries compared to others. Looking at differences within a specific country in a well identified setting eliminates biases due to different social structures, health care systems, specific policies and measures, and testing procedures for the virus that can confound estimates and hinder comparability across countries. Further, we use individual level data as well as Apple mobility data to investigate potential mechanisms. The results highlight the cultural dimension of the spread and could suggest the implementation of targeted mitigation measures in light of disease outbreaks.
\end{abstract}

Keywords: COVID-19; culture; transmission; morbidity; mortality;

JEL codes:

* Department of Economics, City University of London.

†King's Business School, King's College London. 


\section{Introduction}

Since the recent onset of the SARS-CoV-2 outbreak, which resulted into the COVID19 pandemic in November 2019 in Wuhan, China the virus has spread rapidly across the globe. Italy and Spain are the countries in Europe that were hit the earliest and hardest by April 2020, with France following suit, whereas Northern European countries seemed to have performed better at least in the initial phase, with the exception of the UK. As a result, and despite the relatively homogeneous containment measures (e.g. school closures, lock-downs, social distancing etc.), the incidence and death toll vary substantially across countries and it is difficult to uncover the reasons behind this. The timing of both the onset of the pandemic and the policies to constrain it could clearly play a role; for example in Sweden, Belgium or the UK. However, recent evidence suggests that the virus was present in Europe long before it was initially believed, e.g. as early as December 2019 in France (Deslandes et al. (2020). Yet, it spread faster in some countries compared to others, at least in it the initial stage, resulting in vastly overburdened health systems and excess death rates, before most governments enacted their mitigation policies.

An explanation for this phenomenon could be that social norms, lower levels of social interactions and culturally inherent "social distancing" in some Northern societies may have slowed the spread of the virus to vulnerable groups, e.g. the elderly (Bayer and Kuhn (2020)). Recent evidence has demonstrated that disease transmission patterns are crucially characterised by the interactions among people (Alfaro et al. (2020); Liu et al. (2020); Platteau and Verardi (2020)). However, substantial differences in social structures, demographics, health systems, testing, social distancing policies and all other forms of policy responses at different time periods render cross country comparisons rather unhelpful. For this reason we choose to focus on a single country; Germany, where such concerns are rather mitigated.

We observe a substantial discrepancy even at the local authority (NUTS-3) level in per capita incidence and number of deaths between Catholic and non-Catholic regions using daily data from the Robert Koch Institute. We confirm this using Difference-in-Difference estimates at the NUTS-1 level. This could hint at a type of "cultural transmission" which remains strong after partialling out socio-economic characteristics, geographical proximity to Northern Italy, mobility trends before the lockdowns, and regional fixed effects. Catholic regions that arguably exhibit stronger social and family ties seem to experience a wider spread of the virus in the general -as well as the more vulnerable (or elderly) population- as indicated by their higher death toll. Previous research in economics has established that Catholics are more bound to close social circles and networks, e.g. family and friends, and they have different patterns of social interactions (Arruñada (2009); 
Glaeser and Glendon (1998); Ekelund et al. (2002)). This cultural differences in behaviour and social ethics can trigger a differentiated transmission of the disease in the population during early stages. We demonstrate that the COVID-19 incidence varies systematically with religion at the regional level. The results suggest that culture leads to different transmission rates within groups. The proposed mechanism is supported by results using individual-level data from the European Social Survey (ESS), the European Values Survey (EVS) and Apple mobility data that help us investigate how Catholics are systematically and culturally different in their social and family ties, and hence uncover a potential disease transmission channel within this group.

These results could support epidemiologists and public health policy makers to better understand how cultural factors can largely influence the spread of pathogens in a society, and account for these differences when designing response policies as suggested by Platteau and Verardi (2020). The remainder of the paper is organized as follows: In Section 2 we present an overview of the pandemic and policy response in Germany. Section 3 describes our identification strategy, data and results, whereas Section 4 discusses and concludes.

\section{Background}

According to data released from the Robert Koch Institute (RKI), the first reported cases of COVID-19 in Germany were recorded on 27 January 2020 in Starnberg, Bavaria and by March all German states had reported cases. The first deaths were reported on 9 March in North Rhine-Westphalia and by the end of the month all states confirmed hospitalized virus-related deaths. State and federal government response was swift (Stafford (2020)). In early March the federal government and the RKI issued the National Pandemic Plan to be carried out across the country. The states themselves were given some autonomy in handling the pandemic, although the response was coordinated and crucial to avoid inter-state travel. All states in Germany enacted strict social distancing measures and closures of schools, shops and workplaces. Based on the Coronavirus Government Response Tracker developed by the University of Oxford, these social distancing measures were implemented on February 29 (public events cancellation), March 16 (school closings), March 22 (shops and workplaces closings) and April 09 (public transport closing). The number of daily cases peaked in early April according to the RKI and started receding from that point on. Some of the nationwide restrictions such as the closure of small shops were lifted about three weeks later on 22 April.

In terms of testing, widespread PCR testing was made available on 25 March and restrictions for severe cases were lifted, so that more than 2 million people received a 
test by late April. Data on cases and deaths are collected by local authorities (NUTS-3), reported to the Federal Ministry of Health and published by RKI after validation. As of 23 April 2020, 148,046 confirmed cases were reported as well as 5,094 deaths. Figure A.1 displays how the cumulative numbers of reported cases and deaths per 100,000 population were scattered across the country on April 20.

This is the period we will focus on, as the initial stages of the pandemic largely determine the speed of the spread (Zhao et al. (2020)). Given the relatively long incubation period of up to two weeks (Lauer et al. (2020)) and the large number of asymptomatic carriers (Gudbjartsson et al. (2020), the virus can remain undetected and spread faster in the general population during the early stage before mitigating strategies can come to full effect.

\section{Empirical analysis}

\subsection{Identification Strategy}

Given the large number of factors that influence the spread and toll of the disease, it is difficult to compare across countries. However, policy responses have been rather similar resulting in arguably varying degrees of efficiency. This can be to some extent explained by differences in societal and individual behaviour and attitudes rather than different approaches in testing or capabilities of health care systems. Yet, our purpose is to determine whether cultural aspects played a role.

We choose to focus within a single country on a religious divide that has been used in the literature before to explain how cultural differences may affect individual behaviour (Iannaccone (1998); Ekelund et al. (2002); Arruñada (2009); Spenkuch (2017); Spenkuch and Tillmann (2018); Becker and Pascali (2019)). Specifically, we observe that in Germany regions with a higher share of Catholics seem to have been more severely affected by the virus, which could be explained by stronger social and family ties. ${ }^{1}$ For example, when looking Figure A.1 in the Appendix, there is a striking similarity between how the disease has spread and how Catholics are scattered across regions.

Moreover, in 1 the spread of the disease seems to have evolved differently over time in Catholic regions, i.e. where Catholics are the majority, versus non-Catholic ones. In robustness checks, this is evident regardless how Catholic regions are defined. More specifically, a NUTS-3 region is defined as a Catholic (vs. a non-Catholic one) if: (a) Catholics are more than Protestants in that region; (b) Catholics are the majority in that

\footnotetext{
${ }^{1}$ We choose to focus on West Germany only, as the East part historically exhibits systematic differences and could bias our results (Becker et al. (2020)). Given the very low number of cases/deaths and Catholics it would very likely bias the results in our favour.
} 
region; (c) the share of Catholics in that region is higher than their national average; and (d) the share of Catholics in that region is higher than their share in the respective NUTS-1 area. The spread of the virus at the onset of the pandemic is higher in NUTS-3 regions dominated by Catholics, and this led to higher levels of reported COVID-19 cases per 100,000 population in those areas, as well as a higher number of resulting deaths.

[Figure 1 here]

To clearly identify this cultural aspect we look as to whether there were any differences between past overall mortality in Catholic and non-Catholic regions similar to a difference-in-difference pre-trends design (Appendix Figure A.2). Time series begin in 2011, considerably after the last major swine flu outbreak in 2009. To our knowledge, no other major threat to public health that can be transmitted via social contacts occurred since then. Both lines are quite close and move in parallel implying that until the COVID-19 pandemic nothing noticeable caused Catholics to pass at a higher rate, relative to non-Catholics. Any systematic differences in genetic predisposition, socioeconomic characteristics or behaviours such as risky attitudes would likely reflect in differences in mortality rates between groups in the past. Therefore, we are confident that it is cultural aspects on the disease transmission that we are looking at. Several other demographic and economic characteristics are also balanced between Catholic and non-Catholic regions before the pandemic as can be seen in Table A.1 in the Appendix. ${ }^{2}$

A threat to identification could be that two of the most prominent Catholic states (Bavaria and Baden-Württemberg) are in closer geographical proximity to Northern Italy, which was reportedly the first European area hit the hardest by the pandemic. Even though large airports are the primary entryways into the country, we consider land travel as well as some recent working papers suggest (Pluemper and Neumayer (2020)). To that end, we use Google Maps data, to also check whether driving distance from Milan, Italy, affects our baseline estimates. To further argue about the cultural transmission rather than geographic proximity, we additionally focus on North Rhine-Westphalia, a state further away from Germany's southern border and one in which large shares of Catholics and non-Catholics reside. Some early new articles as well as working papers (Pluemper and Neumayer (2020)) suggest that the higher spread in this state is likely to be because of numerous carnival festivities in February. To address this concern, we look at Apple daily mobility data.

\footnotetext{
${ }^{2}$ For example, defining Catholic regions as those where Catholics are the majority, there are no notable differences in characteristics like share of foreigners, mean age, hospital beds per person, GDP per capita, and share of people over 65 years old. Protestant areas have higher unemployment, more people completed secondary education and they are more densely populated.
} 
These data are obtained through GPS tracking and they are available at the city level (after 13 January 2020, and relative to that date). They provide information regarding requests for directions by transport type, i.e. walking, driving and transit. We classified the cities available for (West) Germany into Catholic- and non-Catholic dominated ones and we calculated the mean mobility indicators for each group of cities (weighted by the local population). Trends by transportation type before and after the lock-down (22 March, 2020) are in Figure 1. There is a spike in using public transport about 30 days before the lockdown, which coincides with the culmination of carnival festivities in late February. However, this spike is visible not only in Catholic, but also in nonCatholic cities during that period, and the two lines (just as the spread per capita) are indistinguishable from each other. Moreover, even though carnival started as a Catholic tradition, it has evolved into a nation-wide celebration for the youth, so that transmission of an infectious disease is just as likely anywhere in the country.

A natural starting point for the analysis is to demonstrate that the reported COVID19 incidence does vary with religion at the regional level. This is challenging because demographic information at a geographically disaggregated level do not arrive at the same frequency as data on infectious diseases do. Nevertheless, we model the number of COVID-19 cases as follows:

$$
Y_{r d}=\alpha Y_{r d-\tau} S_{r d-\tau}+\beta \text { Catholic }_{r}+X_{r} \gamma+t_{r}+\lambda_{R}+\epsilon_{r d}
$$

where $Y$ is the number of reported COVID-19 cases in NUTS3 region $r \in\{1, \ldots, N\}$ in day $d$ of the outbreak, Catholic is the logged share of Catholics in region $r$ (based on recent data before the virus outbreak), $t_{r}$ is a linear time trend starting from the day when the first case was reported in each region, $\lambda_{R}$ is a set of regional fixed effects (at a more aggregated level so they are not perfectly collinear with the demographic and economic predictors) and $\epsilon_{r d}$ is the error term.

The variable $S$ represents the proportion of susceptible individuals in the regional population, where their stock in each region has been approximated as the number of people after removing those reported deceased from the virus in each day (Adda (2016)). The $\tau$ parameter represents the incubation period and has been set equal to 14 days, although we results are robust to a wide range of alternative lags (i.e. 3 to 20 days). (Lauer et al. (2020)). We do not consider any spatial variation in the model so the incidence rate in each region is solely determined by its own past realisations, i.e. parameter $\alpha$ should be interpreted as an estimate of the within-region spread. All models control for the size of the local population and for a series for economic and demographic controls, as well as for travelling distance from Milan. Estimating these empirical models will provide an indication of whether the spread of the COVID-19 disease varies with the 
share of Catholics in the region, conditional on other characteristics, area fixed effects and regional time trends.

A problem with this specification is that regional (at the NUTS-3 level) time-invariant unobserved heterogeneity is not adequately partialled out; although results are conditional to broader-level regional fixed effects. To address this issue, we apply the two-step method suggested by Pesaran and Zhou (2018) in order to uncover time-invariant effects in a case where $N$ is large $T$ is small and fixed. More specifically, the predicted residuals from a fixed-effect estimation are averaged for each NUTS-3 region over the entire period. In the second step, they are used as the dependent variable in an OLS regression over the cross-sectional sample of NUTS-3 regions, where models control for the share of Catholics and other local characteristics (e.g. travelling distance from Milan and socio-economic variables). The results are robust even after taking out the regional fixed effect, indicating a positive relationship between the virus spread and the prevalence of Catholics in the region.

Our main hypothesis is whether the difference mortality in Catholic versus nonCatholic regions is higher after the onset of the pandemic. Having established that the pre-pandemic mortality trends were similar in both regions (Figure A.2 in the Appendix), we empirically test for this by adopting the following difference-in-differences framework:

$$
m_{r t}=\beta_{0}+\beta_{1} \text { Catholic }_{r}+\beta_{2} \text { Onset }_{t}+\beta_{3} \text { Catholic }_{r} \times \text { Onset }_{t}+u_{r t}
$$

where $m_{r t}$ is the mortality rate in the $r$-th region in day $t$, Catholic is an indicator of a predominantly Catholic region and Onset is a dummy variable switched on after the first COVID-19 related death in the region was reported. The coefficient of interest in Equation 2 is $\beta_{3}$ and indicates whether differences in mortality rates between Catholic and non-Catholic regions have changed after the onset of the pandemic and as a result of it. Based on our hypothesis about greater transmission of the disease in regions where social ties are stronger, we should expect that $\beta_{3}>0$.

\subsection{Data}

We combine several data sources. Data on COVID-19 cases come from the Robert Koch Institute (RKI) which is a German federal government research institute responsible for disease control and prevention. It has been publishing validated data on reported COVID19 cases and related deaths since January 28, 2020. These are daily data by gender, age group and administrative district (412 Landkreise in total). In this analysis, we focus only on the 324 West Germany districts, because including East Germany could bias the results (Becker et al. (2020)). In order to be matched to a series of regional characteristics, 
these COVID-19 series were collapsed by NUTS-3 region and date (running from January 28 to May 01), resulting in a balanced panel of more than 32,000 observations.

Our main controls are taken from the Federal Statistical Office (Statistisches Bundesamt of Germany and are validated until 2017. These include a number of demographic and socioeconomic characteristics at the regional level, e.g. population and population density, share of people over 65 years old, share of foreigners, share of males and females, share of those who completed secondary education, GDP, number of hospital beds, number of nights spend per person as an indicator for tourism-related activity, and number of vehicles per capita (motorisation rate) as a proxy for travelling and commuting intensity in each region. Moreover, we use Google Maps data in order to calculate the fastest driving distance between Milan, Italy and the major city of each NUTS-3 region. We also use the 2011 German Census in order to calculate the number of Catholics, Evangelicals and other/no denominations in each region. The share of Catholics relative to the total population in the area will be our main variable of interest.

The individual level data stem from the European Social Survey (ESS) 2018 and the European Values Survey (EVS) 2018, two representative data sets of 1,881 and 4,259 adults in West Germany respectively, covering a range of questions around values and social norms. The variables of interest proxy for close family and social ties, i.e. the frequency of social interactions, the perceived frequency compared to individual of the same age, the number of people one is comfortable confiding in, the importance of family, the importance of friends, the level of trust towards family members and whether the respondents reside with their parents or parents-in-law. Further, we use individual and household characteristics as controls. These include the age of the respondent, gender, employment status, education level, subjective health status, age of the youngest household member (EVS only), household size, household income, size of the city/town (EVS only) and NUTS-1 fixed effects. Finally, to check for differences in mobility, we use Apple daily data on mobility volume (by means of transportation) at the city level.

\subsection{Results}

\subsubsection{Baseline results}

Table 1 displays the results from Equation 1 using the daily number of new reported COVID-19 cases per NUTS-3 region as outcome. To address any issues regarding proximity to the Northern Italian border, we used Google Maps to calculate the fastest driving distances from Milan, Italy to the major city of each one of the West German NUTS-3 regions. Although this does not completely rule out any geographic heterogeneity, it mitigates any concerns with respect to geographic dispersion of the pandemic in Europe. 
In Column 1 we use our full set of controls including travel distance to Milan and state fixed effects and we observe a positive and statistically significant relationship between the local share of Catholics and COVID-19 incidence. The 14-day lagged COVID-19 incidence is as expected a strong predictor of today's count. ${ }^{3}$

[Table 1 here]

The same conclusions hold when the cumulative number of reported COVID-19 cases is considered as the outcome, in Appendix Table A.2. Again, the lagged number of cumulative incidence at the regional level is a strong determinant of the current spread, although to a lesser extent. Hence, the results in Column 4 of 1 should be expected, i.e. a positive relationship between the local share of Catholics and the number of deaths, as well as the cumulative number of deaths (Table A.2 Column 4). ${ }^{4}$

The three states with most cases and deaths as of April 2020 are Bayern, BadenWürttemberg and North Rhine-Westphalia. The former two are predominantly catholic, $56 \%$ and 38\% Catholics, respectively, whereas North Rhine-Westphalia is split in the middle. However, the two southern states of Bayern and Baden-Württemberg border on Austria and are geographically closer to Northern Italy, the alleged epicenter of the pandemic in Europe. As this geographical proximity might affect our results, we focus part of the analysis on North Rhine-Westphalia where the share of Catholics is high at $42 \%$, but a substantial share of Evangelicals (28\%) also live there. When the estimation sample is restricted to that state, in Column 3 of Table 1, a positive relationship still emerges after controlling for time effects and local demographics. This is also the case when the outcome is the number of deaths, in Column 6 of Table 1. With respect to the cumulative number of cases in Column 3 of A.2, the relationship is not statistically significant, but remains positive and in line with what reported when conditioning on all West Germany regions. However, it is again positive and highly significant when considering the cumulative number of deaths in Column 6 .

All these results are conditional to greater region fixed effects, because controlling for NUTS-3 fixed effects in the baseline models would not allow to obtain estimates for the local Catholics share. However, this could produce biased coefficients, i.e. in the case where the share of Catholics (and the spread of the virus) are correlated with timeinvariant unobserved heterogeneity at the regional level. This is a common problem in

\footnotetext{
${ }^{3}$ The 14-day lagged number of incidence has been multiplied by a factor that represents the fraction of susceptible individuals in the local (NUTS-3) population. This factor is an approximation, and the local population is calculated as the population minus the cumulative number of COVID-19-related deaths in each day. As such, it does not consider population changes due to local-specific fertility and mortality from other causes, and does not address any endogeneity concerns. Nevertheless, results are robust to the inclusion of various lags, ranging from 3 to 20 days.

${ }^{4}$ In all cases the results are robust to the inclusion of day-of-week fixed effects to account for constant differences in working and leisure patterns across localities.
} 
the emerging COVID-19 empirical literature because regional economic and demographic covariates are not available at the daily level and during the (recent) period under study.

Therefore, as an attempt to address this issue, we use the fixed-effects filtered (FEF) estimator suggested by Pesaran and Zhou (2018) in order to uncover the effect of a time-invariant covariates when using a panel dataset where $N$ is large and $T$ is small. More specifically, in the first step the (logged) number of COVID-19 cases (and deaths) was regressed on a set of NUTS-3 fixed effects, past COVID-19 incidence, day of week fixed effects and a regional linear time trend (fixed effect estimation). Then, the residual COVID-19 incidence (and related deaths) was obtained and averaged over the period for each region. In the second step, we regressed this mean residual on the local Catholics share and other local characteristics using the cross-sectional sample of NUTS-3 regions. The results, in panel A of Table 2, confirm a positive and significant relationship. Moreover, the estimated coefficients remain positive and significant even after controlling for demographic and economic characteristics and driving distance from Milan. The picture remains largely the same when repeating the same exercise for the number of deaths.

[Table 2 here]

Moreover, we estimate whether the spread of the virus was higher in two phases of the pandemic in Germany, i.e. before and after the general lockdown implemented on March 22, 2020. The results are in Table 2, panel B. In this case, we estimated the first step residuals using data only until the implementation of the lockdown. The effect of Catholics share remains positive and significant on regional characteristics. Then we use the COVID-19 data to estimate residual virus incidence in each region during the lockdown period. The local share of Catholics remains a positive and significant predictor even after controlling for local characteristics and the $(\log )$ stock of cumulative cases per 100,000 local population at the date of lockdown implementation. This suggests that after mobility was restricted and filtering out the already existing number of cases, transmission was still higher among Catholics.

We then proceed with a difference-in-differences estimation using the total number of deaths from all causes across regions. A limitation is that only NUTS-1 level counts are provided by the Federal Statistical Office. The time series span from 1 January until 17 May. NUTS-1 West Germany regions are split into predominantly Catholic vs. nonCatholic. To capture the "after" dimension we consider the number of deaths after the first reported death in each region. The results are in column 1, Table 3. We then proceed to limit the time span until 22 April which is when some restrictions were lifted and it is roughly a month in since the country entered the lockdown. This would allow to capture 
fatalities resulting from the initial stage of the pandemic ${ }^{5}$. In Column 3 we add a time trend (days since first reported death) to potentially capture the already higher spread in some regions compared to others. Further, we add NUTS-1 fixed effects in Column 4. Finally, we change the time variable to "first reported case" in Column 5 to capture the already existing high cases counts before the first fatalities were reported. In all of our estimation the difference-in-difference estimator is positive and highly significant ensuring that both the spread and fatalities were higher in predominantly Catholic regions.

\subsubsection{Mechanisms}

Having established a positive link between COVID-19 incidence and Catholics share at the local level, a possible mechanism could operate through social norms, i.e. tighter social networks and stronger family ties. Our aim is not to discuss religion or Catholicism in Europe in itself, but rather to explore whether cultural differences within a very similar population could have impacted groups differently.

Historically, it seems that Protestants and non-Catholics have developed more market oriented and individualistic behaviours (Becker and Pascali (2019)), whereas catholic regions were characterized by rent-seeking behaviours through established social structures (Ekelund et al. (2002)). Moreover, Arruñada (2009) argues that these differences are mostly prominent in social dimensions and ethics and that social interaction is regarded by Protestants only as an enforcement and control mechanism. This implies that social interaction as an intrinsic behaviour is more likely to be found among Catholics. For these reasons we believe that Catholics in Germany exhibit stronger social and family ties.

To look deeper into potential mechanisms, we turn to the European Social Survey 2018 (Wave 9) for Germany. The survey contains questions on an individual level on the frequency of social interactions, on the perceived frequency of such interaction compared to ones peers and also a question on the number of individuals with whom one feels comfortable discussing private and individual matters. We code the first two questions as dummies indicating the frequency of social interactions as more than "several times a week" and the perceived relative measure as "at least the same". The third variable of interest is the number of individuals respondents confide in. Further, we control for age, gender, subjective health status, employment status, household size, household income and NUTS-1 fixed effects. We also code a dummy for identifying as belonging to the Catholic denomination. The results in Section A of 4 suggest that there is a positive relationship between belonging to the Catholic denomination and our measures for social

\footnotetext{
${ }^{5}$ The results are robust to limiting the time-frame until 31 March which would still capture infections prior to the lockdown
} 
interactions. The coefficient for frequency is barely not significant at the $10 \%$ level (tstat $=1.67$ ), but the other two are, indicating that Catholics tend to have more frequent social interactions and exhibit stronger ties.

The 2018 European Values Survey provides another set of questions that could serve as proxies for these attitudes and behaviours. Here we employ a set of regressions using individual-level data on the importance of family and friends in an individual's life, the level of trust towards members of the family and the likelihood to reside with parents (or parents-in-law) in the same household. Our variable of interest is simply a dummy on whether the individual belongs to the Catholic denomination. Controlling for the same set of socioeconomic characteristics and regional fixed effects as in Section $\mathrm{A}^{6}$, the results in Section B of Table 4 indicate that Catholics, relative to non-Catholics, regard their family and friends as very important, exhibit higher trust towards family members and have a higher probability of residing in the same household with their parents or parents-in-law. This is in line with Arruñada (2009) and suggests that Catholics exhibit much stronger social and family ties, which in turn could be a mechanism through which a higher spread and incidence of COVID-19 among Catholics operates.

To further strengthen our proposed mechanism, we employ another set of regressions with the individual-level data. The idea is to determine whether cultural differences could affect the differential outcomes of the outbreak in other ways rather than through social contacts. Specifically, we test whether Catholics have a higher propensity to justify cheating behaviour in avoiding taxes, accepting bribes and avoiding fares in public transport. Further, we examine their confidence in the government, even though recent research suggest that in most European countries confidence in the governments and the implemented measures have increased (Bol et al. (2020)) and that during the recent pandemic individuals tend to prioritize health (Hargreaves Heap et al. (2020)). This should indicate whether the higher spread among Catholics could be a result of overall riskier behaviour or groups disregarding the rules and the protective measures put into place. This would likely have a differential impact on the spread and mortality of the disease after the measures came into place. We empirically test this hypothesis using individual-level German data from the European Values Survey.

Broadly speaking, the data reveal that generally adherence to rules is very high in Germany. Moreover, the regression results in Section $\mathrm{C}$ of Table 4 do not show a higher propensity for Catholics to disobey rules using the same set of individual controls as in Section A and regional fixed effects. If anything, Catholics are less likely to tolerate even the milder offense (avoiding fare) and exhibit marginally higher confidence in the

\footnotetext{
${ }^{6}$ Additionally we control for the age of the youngest household member and the size of the city, which are only provided in the EVS
} 
government. For this reason we believe that the increased spread is unlikely to be a result of risky behaviour and disregard for rules or the government's measures and more related to social and family networks.

To further rule out other behavioural differences between Catholics and non-Catholics, we look again at the Apple mobility data in Figure ??. There is a significant drop in mobility after the lockdown. However, there is no significant differentiation on the basis of religion. We are therefore confident that the higher transmission rates among Catholics are likely to be a results of close social circles and strong family ties and not of higher mobility after the lockdown.

[Table 4 here]

\section{Discussion}

Culture shapes social norms and, to a large extent, dictates societal and individual behaviour. Cultural differences are frequently unobserved. Nevertheless, it is of very high importance to take them under consideration as they can lead to different outcomes. Such heterogeneities need to be taken into account not only in epidemiological modelling and parametrization, but also to design optimal policy responses. These heterogeneities do not only exist between, but also within countries and often go beyond standard socioeconomic characteristics.

This is all the more important when facing crises such as the current COVID-19 pandemic. Being able to explain the wildly different trajectories of countries could provide invaluable insight to policymakers to better target mitigation measures and policies around pandemics (Platteau and Verardi (2020)). Previous attempts to highlight the importance of culture and how it can determine social interactions are marred by unobserved heterogeneity (Bayer and Kuhn (2020)) and it is difficult to disentangle the number of factors that need to be taken into account. Differences in the timing of the pandemic, health care systems, testing methods to accumulate data and the timing and the nature of policy response, render cross country comparisons rather difficult.

In this paper we used data on daily cases and deaths attributed to COVID-19 in West Germany to overcome some of these issues and to identify the impact of cultural differences on the spread of the disease and the resulting death toll. Our results suggest that Catholics that inherently exhibit stronger social and family networks were more severely affected. This could explain why in societies with stronger social- and family ties such as Italy and Spain the spread was rapid from the onset and ultimately lead to a high death toll in the initial phase of the outbreak. 


\section{References}

ADDA, J. (2016): "Economic activity and the spread of viral diseases: Evidence from high frequency data," Quarterly Journal of Economics, 131, 891-941.

Alfaro, L., E. Faia, L. N., And S. F. (2020): "Social Interactios in Pandemis: Fear, Altruism and Reciprocity," NBER Working Paper, 27134.

ArruñadA, B. (2009): "Protestants and Catholics: Similar work ethic, different social ethic," Economic Journal, 120, 890-918.

BAYer, C. AND M. Kuhn (2020): "Intergenerational ties and case fatality rates: A cross-country analysis," CEPR, Working Paper.

Becker, S. And L. Pascali (2019): "Religion, Division of Labor, and Conflict: AntiSemitism in Germany over 600 Years," American Economic Review, 109, 1764-1804.

Becker, S. O., L. Mergele, And L. Woessmann (2020): "The Separation and Reunification of Germany: Rethinking a Natural Experiment Interpretation of the Enduring Effects of Communism," Journal of Economic Perspectives, 34, 143-171.

Bol, D., M. Giani, A. Blais, And P. J. Loewen (2020): "The effect of COVID-19 lockdowns on political support: Some good news for democracy?" European Journal of Political Research.

Deslandes, A., V. Berti, Y. Tandjaoui-lambotte, C. Alloui, E. M. CarBonnelle, J. Zahar, S. Brinchler, And Y. Cohen (2020): "SARS-COV-2 was already spreading in France in late December 2019," International Journal of Antimicrobial Agents, ., .

Ekelund, R., R. HÉBert, And R. Tollison (2002): "An Economic Analysis of the Protestant Reformation," Journal of Political Economy, 110, 646-671.

Glaeser, E. AND S. Glendon (1998): "Incentives, predestination and free will," Economic Inquiry, 36, 429-443.

Gudbjartsson, D., A. Helgason, H. Jonsson, O. Magnusson, P. Melsted, G. Norddahl, J. Saemundsdottir, A. Sigurdsson, P. Sulem, A. AgustsdotTIR, B. ERIKSDottir, AND R. E. Fridriksdottir (2020): "Spread of SARS-CoV-2 in the Icelandic Population," New England Journal of Medicine. 
Hargreaves Heap, S., C. Koop, K. Matakos, A. Unan, and N. Weber (2020): "COVID-19 and People's Health-Wealth Preferences: Information Effects and Policy Implications," SSRN Working paper.

IANNACCONE, L. (1998): "Introduction to the Economics of Religion," Journal of Economic Literature, 36, 1465-1495.

Lauer, S., K. Grantz, O. Bi, F. Jones, Q. Zheng, H. Meredith, A. Azman, N. REICH, AND J. LESSLER (2020): "The incubation period of coronavirus disease 2019 (COVID-19) from publicly reported confirmed cases: Estimation and application," Annals of Internal Medicine, ., .

Liu, Y., Z. Gu, S. Xia, B. Shi, X. Zhou, Y. Shi, And J. Liu (2020): "What are the underlying transmission patterns of COVID-19 outbreak? An age-specific social contact characterization," EClinicalMedicine, ., .

Pesaran, M. and Q. Zhou (2018): "Estimation of time-invariant effects in static panel data models," Econometric Reviews, 37, 1137-1171.

Platteau, J.-P. And V. Verardi (2020): "How To Exit Covid-19 Lockdowns: Culture Matters," CEPR, Covid Economics, 23, 1-57.

Pluemper, T. And E. Neumayer (2020): "The COVID-19 pandemic predominantly hits poor neighborhoods, or does it? Evidence from Germany," medRxiv 2020.05.18.20105395.

Spenkuch, J. And P. Tillmann (2018): "Elite Influence? Religion and the Electoral Success of the Nazis," American Journal of Political Science, 62, 19-36.

SpenkuCH, J. L. (2017): "Religion and work: Micro evidence from contemporary Germany," Journal of Economic Behaviour and Organization, 135, 193-214.

Stafford, N. (2020): "Covid-19: Why Germany's case fatality rate seems so low," $B M J$, Opinion.

Zhao, S., Q. Lin, J. Ran, S. Musa, G. Yang, W. Wang, Y. Lou, D. Gao, L. YANG, D. He, AND M. WANG (2020): "Preliminary Estimation of the Basic Reproduction Number of Novel Coronavirus (2019-nCoV) in China, From 2019 to 2020: A Data-Driven Analysis in the Early Phase of the Outbreak," Int J Infect Dis, $92,214-217$. 


\section{Figures and Tables}

Figure 1: Spread of COVID-19 and mobility in Catholic and non-Catholic regions.
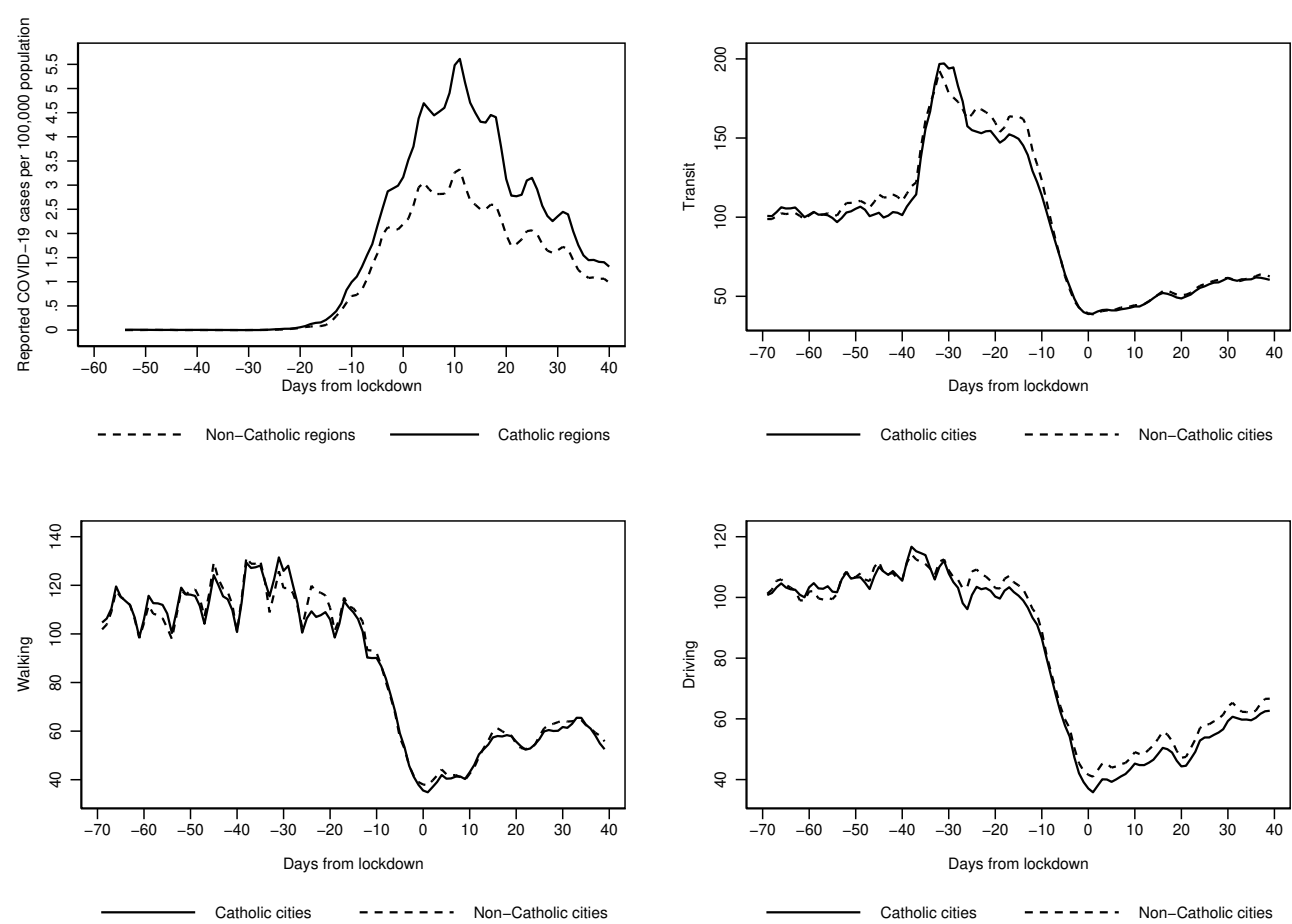

Source: Robert Koch Institute; Apple. Catholic regions and cities are defined as those where Catholics are a majority. Horizontal axis is centered at the lockdown date (22 March, 2020). Relative Apple mobility volumes are weighted by the local population. 
Table 1: Number of reported COVID-19 cases/deaths and local Catholics share in West Germany.

\begin{tabular}{lcccc}
\hline \hline & Cases & Cases & Deaths & Deaths \\
& {$[1]$} & {$[2]$} & {$[3]$} & {$[4]$} \\
\hline \% Catholics & $.153^{* * *}$ & $.105^{*}$ & $.339^{* * *}$ & $.321^{*}$ \\
Lagged cases & $(.045)$ & $(.058)$ & $(.098)$ & $(.182)$ \\
& $.414^{* * *}$ & $.334^{* * *}$ & $.656^{* * *}$ & $.623^{* * *}$ \\
Daily trend & $(.016)$ & $(.024)$ & $(.044)$ & $(.090)$ \\
Local controls & Yes & Yes & Yes & Yes \\
State FE & Yes & Yes & Yes & Yes \\
Sample & Yes & Yes & No & Yes \\
Observations & WG & NRW & WG & NRW \\
NUTS-3 regions & 16,952 & 2,938 & 16,952 & 2,938 \\
\hline
\end{tabular}

Source: Robert Koch Institute (RKI). Gamma regression estimates. Standard errors in parentheses are clustered by NUTS-3 region. WG denotes West Germany and NRW denotes North Rhine-Westphalia.

Table 2: Residual of fixed-effects regressions and local Catholics share in West Germany: Estimates before and after the lockdown.

\begin{tabular}{|c|c|c|c|c|c|}
\hline Panel A: Total period: & & Cases & Cases & Deaths & Deaths \\
\hline \multirow[t]{2}{*}{ \% Catholics } & & $.156^{* * *}$ & $.113^{* * *}$ & $.019^{* * *}$ & $.013^{* *}$ \\
\hline & & $(.022)$ & $(.020)$ & $(.004)$ & $(.006)$ \\
\hline R-squared & & .133 & .589 & .043 & .151 \\
\hline Local controls & & No & Yes & No & Yes \\
\hline Observations & & 312 & 312 & 312 & 312 \\
\hline Panel B: Sub-periods (cases): & \multicolumn{2}{|c|}{ Before lockdown } & \multicolumn{3}{|c|}{ After lockdown } \\
\hline \multirow[t]{2}{*}{$\%$ Catholics } & $.135^{* * *}$ & $.088^{* * *}$ & $202^{* * *}$ & $.133^{* *}$ & $.109^{* * *}$ \\
\hline & $(.029)$ & $(.029)$ & $(.028)$ & $(.026)$ & $(.026)$ \\
\hline Cumulative cases per population & - & - & - & - & $147^{* * *}$ \\
\hline B-sauared & 050 & 565 & 130 & 580 & $\begin{array}{c}(.031) \\
605\end{array}$ \\
\hline Local controls & No & Yes & No & Yes & Yes \\
\hline Observations & 312 & 312 & 312 & 312 & 312 \\
\hline
\end{tabular}

Source: Robert Koch Institute (RKI). The dependent variable is the mean residual of a fixedeffects estimation in the first step. Robust standard errors in parentheses. March 22, 2020 defines the before \& after the lockdown. 
Table 3: Difference-in-difference estimates on the total number of deaths in West Germany at the NUTS-1 level

\begin{tabular}{lccccc}
\hline \hline & Deaths & Deaths & Deaths & Deaths & Deaths \\
\hline Diff-in-Diff estimate & $33.686^{* *}$ & $60.024^{* * *}$ & $45.623^{* *}$ & $18.190^{* * *}$ & $12.783^{* * *}$ \\
& $(16.790)$ & $(21.242)$ & $(21.825)$ & $(3.001)$ & $(2.374)$ \\
Until 22 April & No & Yes & Yes & Yes & Yes \\
Days since first death & No & No & Yes & Yes & Yes \\
Regional fixed effects & No & No & No & Yes & Yes \\
Timeline since first case & No & No & No & No & Yes \\
Observations & 1,380 & 1,090 & 1,090 & 1,090 & 1,090 \\
\hline
\end{tabular}

Note: NUTS-1 regions Schleswig-Holstein, Hamburg, Bremen, Niedersachsen and Hessen classified as non-Catholic. Daily number of deaths from all causes since 1 January. The time variable indicates the date of the first reported COVID-19 related death in the state. In the last Column 5 the time variable indicates the first reported case. 
Table 4: Importance of social and family ties and adherence to rules

\begin{tabular}{lcccc}
\hline \hline Section A: Social Interactions & & Frequency & Relative & Closeness \\
\hline Catholic & & 0.044 & $0.056^{*}$ & $0.156^{* * *}$ \\
& & $(0.027)$ & $(0.026)$ & $(0.016)$ \\
Individual Controls & & Yes & Yes & Yes \\
Regional FE & & Yes & Yes & Yes \\
Observations & Family & Friends & Trust & Parents \\
\hline \hline Section B: Social and family ties & $0.040^{* * *}$ & $0.092^{* * *}$ & $0.056^{* * *}$ & $0.017^{* * *}$ \\
\hline Catholic & $(0.011)$ & $(0.022)$ & $(0.017)$ & $(0.007)$ \\
& Yes & Yes & Yes & Yes \\
Individual Controls & Yes & Yes & Yes & Yes \\
Regional FE & 3,247 & 3,237 & 2,338 & 3,247 \\
Observations & Taxes & Bribe & Fare & Government \\
\hline \hline Section C: Adherence to rules & -0.023 & -0.062 & $-0.159^{* *}$ & $0.064^{*}$ \\
\hline Catholic & $(0.058)$ & $(0.064)$ & $(0.064)$ & $(0.034)$ \\
& Yes & Yes & Yes & Yes \\
Individual Controls & Yes & Yes & Yes & Yes \\
Regional FE & 2,322 & 2,333 & 2,333 & 2,269 \\
Observations & & & & \\
& & & &
\end{tabular}

Note: OLS regressions using robust standard errors clustered at NUTS-1 level. The data in Section A are from the European Social Survey 2018 for Germany. The independent variable in the first Column is dummy coded one if the individual meets with others more than "several times a week". In the second Column the dummy takes the value one if the individual perceives themselves to meet more than "about the same" compared to their peers. In the final Column respondents indicate the number of people with whom they "can discuss intimate and private matters". The data In Sections B and C are from the European Values Survey 2018 for Germany. The independent variables in Section B are on a 1-4 scale on the importance of family and friends in ones life and the level of trust towards members of the family, as well as a binary variable on whether one resides with their parents or parents-in-law. The independent variables in Section C are on a 1-10 scale whether it is justified to avoid taxes, to accept a bribe and to avoid fare in public transport, as well as a 4-point scale indicating confidence in the government. We control for perceived health status, gender, age, household income, education level, employment status, age of the youngest household member and size of the city, as well as state fixed effects. 


\section{Appendix}

Figure A.1: COVID-19 spread and local share of Catholics at the NUTS-3 level.
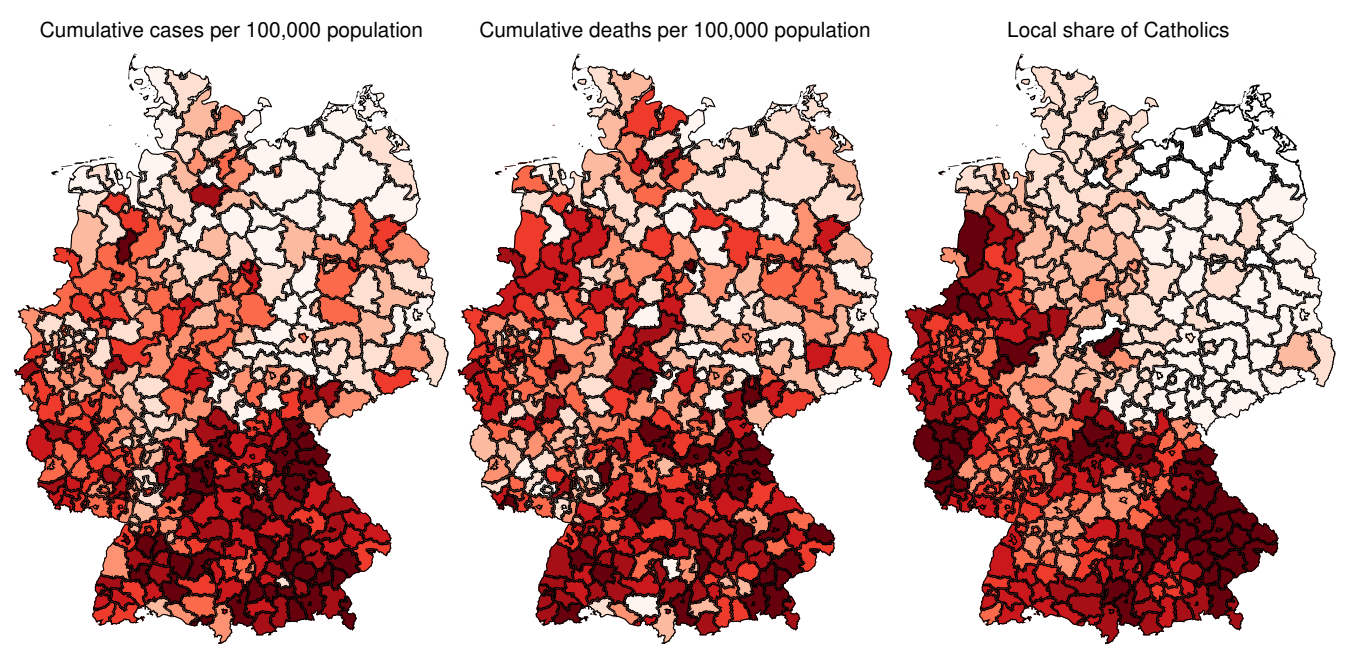

Source: Robert Koch Institute (RKI); Federal Statistical Office of Germany. Notes: Data on COVID-19 spread are as at April 20, 2020. Data on the local share of Catholics refer to 2017.

Table A.1: Means of control variables

\begin{tabular}{lcccccc}
\hline \hline & \multicolumn{3}{c}{ Catholic } & \multicolumn{3}{c}{ Non-Catholic } \\
& Mean & SD & $\mathrm{n}$ & Mean & SD & $\mathrm{n}$ \\
\hline GDP & 11.1597 & .1342 & 162 & 11.1664 & .1573 & 161 \\
Population & 11.9228 & .5842 & 162 & 12.0761 & .7187 & 162 \\
Population Density & 5.5540 & .9298 & 162 & 5.9623 & 1.1596 & 162 \\
Share over 65 & 3.0353 & .0911 & 162 & 3.0714 & .1069 & 162 \\
Foreigners & 2.3109 & .3530 & 162 & 2.3745 & .4660 & 162 \\
Completed Secondary & 3.3753 & .3034 & 160 & 3.4898 & .2541 & 162 \\
Hospital beds per 1,000 & 1.5898 & .7052 & 161 & 1.6871 & .5878 & 159 \\
Vehicles per 1,000 & 6.6520 & .1318 & 162 & 6.5576 & .1648 & 161 \\
Nights spent & .8838 & .2896 & 160 & .8908 & .3136 & 156 \\
\hline
\end{tabular}

Note: All variables have been transformed into natural logarithms. Catholic regions are defined as regions where Catholics are the majority 
Figure A.2: Mortality rate in Catholic and Non-Catholic regions, 2011-2017.

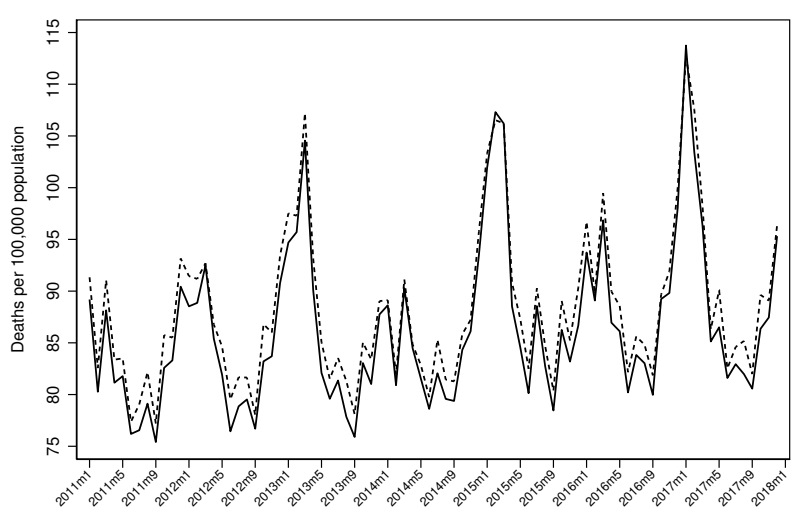

Source: Federal Statistical Office of Germany. Mortality rate is defined as the number of deaths per 100,000 regional population. Catholic regions are defined as those where Catholics are the majority.

Table A.2: Cumulative number of reported COVID-19 cases/deaths and local Catholics share in West Germany.

\begin{tabular}{lcccc}
\hline \hline & Cases & Cases & Deaths & Deaths \\
& {$[1]$} & {$[2]$} & {$[3]$} & {$[4]$} \\
\hline \% Catholics & $.146^{* * *}$ & .111 & $.399^{* * *}$ & $.458^{*}$ \\
Lagged cases & $(.045)$ & $(.085)$ & $(.111)$ & $(.266)$ \\
& $.368^{* * *}$ & $.317^{* * *}$ & $.520^{* * *}$ & $.482^{* * *}$ \\
Daily trend & $(.016)$ & $(.032)$ & $(.027)$ & $(.059)$ \\
Local controls & Yes & Yes & Yes & Yes \\
State FE & Yes & Yes & Yes & Yes \\
Sample & Yes & Yes & No & Yes \\
Observations & WG & NRW & WG & NRW \\
NUTS-3 regions & 16,952 & 2,938 & 16,952 & 2,938 \\
\hline
\end{tabular}

Source: Robert Koch Institute (RKI). Gamma regression estimates. Standard errors in parentheses are clustered by NUTS-3 region. WG denotes West Germany and NRW denotes North Rhine-Westphalia. 